\title{
Rasche Progession, frühe Insulinpflicht
}

Wenn Kinder Diabetes entwickeln, kann man nicht mehr a priori vom Typ 1 ausgehen. Jeder zehnte juvenile Diabetiker in den USA hat Typ-2-Diabetes, oft schon mit Organschäden.

Bezüglich des juvenilen Diabetes wurden auf der ADA-Jahrestagung alarmierende Trends berichtet. Immer mehr Kinder /Jugendliche erkranken an Diabetes: Zwischen 2001 und 2009 wurde ein Anstieg der juvenilen Typ-1-Diabetiker um 23\% sowie der juvenilen Typ-2-Diabetiker um 21\% registriert. Nach Daten der "SEARCH for Diabetes in Youth Study“ wird die Zahl der Kinder/Jugendlichen mit Diabetes in den USA auf 189000 geschätzt, ca. 20000 davon vom Typ 2. Wie Frau Dr. Giuseppina Imperatore aus Atlanta berichtete, haben die Patienten mit so früher Diabetesdiagnose nicht nur ein hohes Risiko, im Lauf des noch langen Lebens Diabeteskomplikationen zu entwickeln. Bei vielen manifestieren sie sich schon im Jugendalter. „Wir fanden bereits nachweisbare Schäden an den peripheren sowie an den autonomen kardiovaskulären Nerven", so die Epidemiologin. Manche Jugendliche, v.a. mit Typ-2-Diabetes, hatten schon eine Proteinurie als Zeichen eines diabetischen Nierenschadens.
Bei Jugendlichen mit Typ-2-Diabetes schreitet die Erkrankung schneller fort, führt früher zu Komplikationen und wird schneller insulinpflichtig. Dies folgt aus einer weiteren, beim ADA vorgestellten Studie, der „Treatment Options for Typ 2 Diabetes in Adolescents and Youth Study“. Darin waren 699 jugendliche Typ-2-Diabetiker im Alter zwischen 10 und 17 Jahren in drei Gruppen behandelt und 2-6 Jahre nachverfolgt worden. „Die gute Nachricht lautet, dass etwa $50 \%$ der Kids früh und gut auf Metformin ansprechen und mit dieser Therapie dann erst einmal stabil eingestellt sind“, berichtete Phil Zeitler, Pädiater an der University of Colorado. Die anderen 50\% müssen bereits nach wenigen Krankheitsjahren mit Metformin plus Insulin behandelt werden. Diese Studie untersuchte zwar Rosiglitazon als Therapiealternative, die heute aber nicht mehr empfohlen werden kann. Laut Zeitler ist es unbedingt nötig, weitere Therapiealternativen für jugendliche Typ-2-Diabetiker zu erforschen.

de

\section{Ist-Status bei Typ-1-Diabetikern}

\section{Schlecht kontrolliert, zu dick, oft Hypos}

\begin{abstract}
Bei Typ-1-Diabetes ist der langfristige prognostische Nutzen einer guten Stoffwechselkontrolle gut dokumentiert. Dennoch sind vor allem Kinder und Jugendliche oft sehr schlecht eingestellt, wie jetzt eine große Register-Studie aus den USA zeigt.
\end{abstract}

Die Typ-1-Diabetes-(T1D-)Inzidenz steigt mit 3\% pro Jahr an. 1 bis 2 der etwa 26 Mio. Diabetiker in den USA haben einen Typ-1-Diabetes, berichtete Dr. Richard Bergenstal von der University of Minnesota. Bei T1D gesichert ist, dass sich eine gute Stoffwechselkontrolle lohnt: Wenn der $\mathrm{HbA}_{1 \mathrm{c}}$-Wert durch intensivierte Insulintherapie von ca. 9\% auf etwa 7\% gesenkt wird, verhindert dies langfristig Komplikationen an Augen, Nieren, Nerven und am HerzKreislauf-System, so das Ergebnis des Diabetes Control und Complications Trial (DCCT und EDIC). Doch offenbar lässt sich diese Erkenntnis in der Praxis schlecht umsetzen, wie jetzt die US-RegisterStudie T1D-Exchange mit 25000 Typ-1-Diabetikern, darunter 13000 Kinder und Jugendliche, zeigt. Die beim ADA 2012 präsentierten Ergebnisse alarmieren.
1. Die Stoffwechselkontrolle ist nicht gut: Kinder bis 13 Jahre haben im Schnitt $\mathrm{HbA}_{1 c}$-Werte von 8,3\%, Jugendliche von $8,6 \%$, und Erwachsene $>26$ Jahre $\mathrm{HbA}_{1 \mathrm{c}}$-Werte von im Schnitt 7,6\%.

2. Die Stoffwechselqualität korrelierte klar mit der Häufigkeit der BZ-Selbstkontrollen. Mit 0-2 Messungen täglich lag der $\mathrm{HbA}_{1 \mathrm{c}}$ bei 9,3\%, mit 3-4 bei $8,5 \%$. Wer täglich mind. $7 \mathrm{mal} \mathrm{maß,} \mathrm{lag}<8 \%$.

3. Adipös/übergewichtig sind $30 \%$ der Kinder mit T1D, 40\% der Adoleszenten, 65\% der Erwachsenen. 4. $4 \%$ (>50 Jahre) und bis 10\% (13-26 Jahre) der Patienten haben jährlich eine Ketoazidose. Schwere Hypoglykämien mit Anfällen/Koma haben 8\% aller Typ-1-Diabetiker mit Krankheitsdauer $<20$ Jahren. Bei längerer T1D-Dauer steigt die jährliche Inzidenz schwerster Hypos auf ca. 20\%. de 